\title{
Explaining sudden losses of outer radiation belt electrons during geomagnetic storms
}

\author{
Drew L. Turner ${ }^{1,2 \star}$, Yuri Shprits ${ }^{1,2,3}$, Michael Hartinger ${ }^{1}$ and Vassilis Angelopoulos ${ }^{1,2}$
}

\begin{abstract}
The Van Allen radiation belts were first discovered in $\mathbf{1 9 5 8}$ by the Explorer series of spacecraft ${ }^{1}$. The dynamic outer belt consists primarily of relativistic electrons trapped by the Earth's magnetic field. Magnetospheric processes driven by the solar wind ${ }^{2}$ cause the electron flux in this belt to fluctuate substantially over timescales ranging from minutes to years ${ }^{3}$. The most dramatic of these events are known as flux 'dropouts' and often occur during geomagnetic storms. During such an event the electron flux can drop by several orders of magnitude in just a few hours ${ }^{4,5}$ and remain low even after a storm has abated. Various solar wind phenomena, including coronal mass ejections and co-rotating interaction regions ${ }^{6}$, can drive storm activity, but several outstanding questions remain concerning dropouts and the precise channels to which outer belt electrons are lost during these events. By analysing data collected at multiple altitudes by the THEMIS, GOES, and NOAAPOES spacecraft, we show that the sudden electron depletion observed during a recent storm's main phase is primarily a result of outward transport rather than loss to the atmosphere.
\end{abstract}

Trapped radiation belt electrons undergo three characteristic types of motion: gyro-motion around magnetic field lines due to any velocity component perpendicular to the field, bounce-motion along field lines between magnetic mirror points due to any velocity component parallel to the field, and drift-motion around the Earth resulting from magnetic gradient and curvature drifts. Associated with each of these oscillatory motions are adiabatic invariants, which are conserved so long as electric and/or magnetic fields do not change on scales similar to those of the associated motions. The first invariant conserves the magnetic moment of the particle and is proportional to the perpendicular momentum squared divided by the local magnetic field strength; the second and third invariants conserve the integral of parallel momentum over one full bounce period and the magnetic flux through a particle's drift orbit, respectively. Magnetospheric changes on timescales much longer than electron drift periods are considered fully adiabatic, that is, they are fully reversible. Initially, it was thought that the observed flux dropouts were fully adiabatic changes in the system. Essentially, electrons moved radially outward (inward) during a storm's main (recovery) phase to conserve their third invariant as Earth's magnetic field was altered by the field produced by an enhanced (weakening) ring current ${ }^{7,8}$, which is a magnetospheric current system resulting from charge-dependent particle drift. As electrons moved radially outward (inward) in the field, their fluxes decreased (increased) for fixed energy as the first adiabatic invariant was also conserved. It was later shown that although this 'Dst effect' (after the disturbance storm time (Dst) geomagnetic index (Kp) used to indicate storm activity) does play a role in the flux dynamics, many flux dropouts do not return to the pre-storm flux level on field recovery. These dropouts therefore result in true particle loss due to irreversible (non-adiabatic) processes $^{9-11}$. Such losses ultimately occur to either the atmosphere or the magnetosphere's outer boundary, the magnetopause ${ }^{12}$. Mechanisms resulting in atmospheric loss include pitch-angle scattering through wave particle interactions with magnetospheric plasma waves ${ }^{13}$ and violation of the first adiabatic invariant due to highly stretched magnetotail fields (that is, those in which the field line curvature becomes comparable to the particle's gyro-radius ${ }^{14}$ ). Several recent statistical studies have concluded that losses to the atmosphere are probably responsible for main phase $\mathrm{e}^{5,11}$ and recovery phase ${ }^{15,16}$ electron loss during certain types of geomagnetic storms. However, the mainphase dropout results were inferred in those studies, with no clear observations showing that the primary loss was to the atmosphere.

Losses to the magnetopause can occur through magnetopause 'shadowing' ${ }^{17}$ and outward radial transport ${ }^{17-19}$. Magnetopause shadowing describes the scenario in which the magnetopause moves inward in response to increased solar wind dynamic pressure and results in the loss of electrons on open drift paths that were closed before the boundary motion. Test-particle simulations have revealed that both enhanced dawn-dusk electric fields ${ }^{19}$ and diamagnetic effects from a partial ring current ${ }^{17}$ during the storm's main phase can result in violation of the third invariant and rapid outward radial transport, resulting in losses to the magnetopause in only a few hours. Radial diffusion can also result in outward transport if the third adiabatic invariant is not conserved and the radial gradient of the electron phase space density (PSD) is negative with respect to increasing radial distance (diffusion always acts to transport particles in the opposite direction to the PSD gradient). Recent studies of PSD radial gradients in the outer belt have shown that most often the gradient is negative for relativistic electrons beyond geosynchronous orbit ${ }^{20,21}$. Also, ultralow-frequency (ULF) waves, a regularly occurring phenomenon, can violate the third invariant condition and allow for electron radial diffusion ${ }^{22}$. Modelling efforts have revealed a scenario combining magnetopause shadowing and outward diffusion as a possible explanation for storm-time outer belt flux dropouts ${ }^{18}$. Most recently, this loss scenario was also proposed to explain observations of an electron dropout event ${ }^{23}$; no evidence discounting loss to the atmosphere was provided, however. Other studies $^{24,25}$ argued that losses could be explained by a combination of magnetopause shadowing and subsequent outward transport at high $L$-shells ( $L>5-6$, with $L$ being the radial distance in $R_{\mathrm{E}}$ (Earth radii) of electron drift orbits in the magnetic equatorial plane) and loss to the atmosphere by interactions with various plasma waves at lower $L$-shells. Ultimately, the long-standing, fundamental question as to what mechanism is primarily responsible for non-adiabatic electron losses during dropout events is still debated.

\footnotetext{
${ }^{1}$ Department of Earth and Space Sciences, University of California, Los Angeles, California 90095-1567, USA, ${ }^{2}$ Institute of Geophysics and Planetary Physics, University of California, Los Angeles, California 90095-1567, USA, ${ }^{3}$ Department of Atmospheric Sciences, University of California, Los Angeles, California 90095-1567, USA. *e-mail: drew.lawson.turner@gmail.com.
} 
a

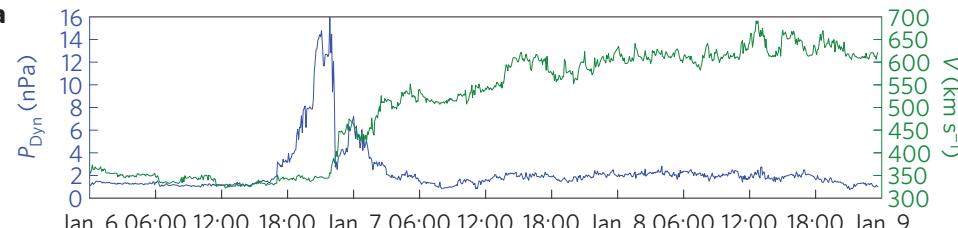

Universal time

b
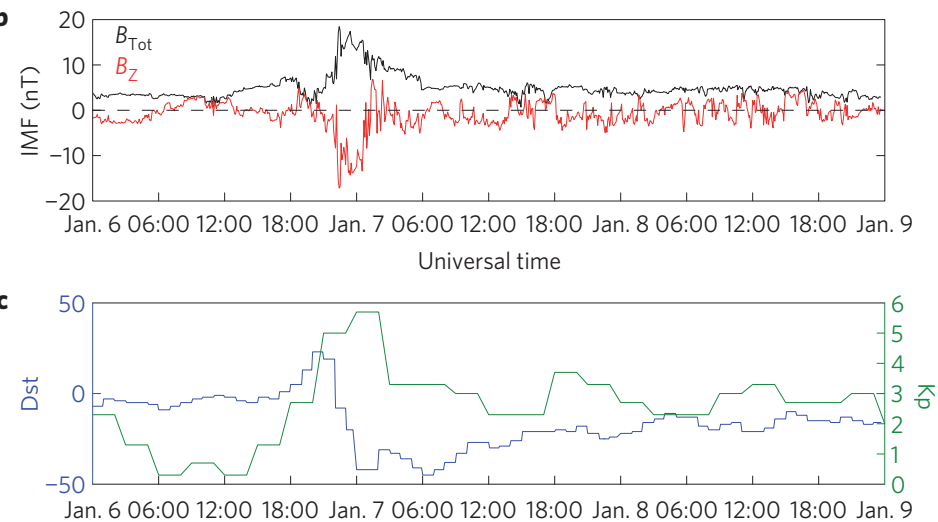

Universal time

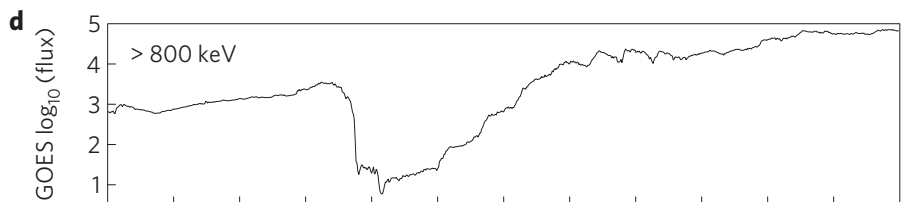

Jan. 6 06:00 12:00 18:00 Jan. 7 06:00 12:00 18:00 Jan. 8 06:00 12:00 18:00 Jan. 9

Universal time

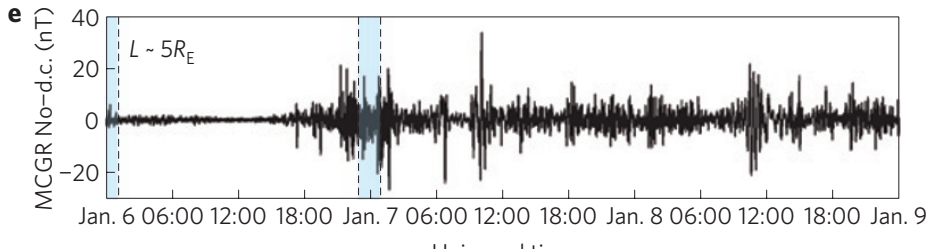

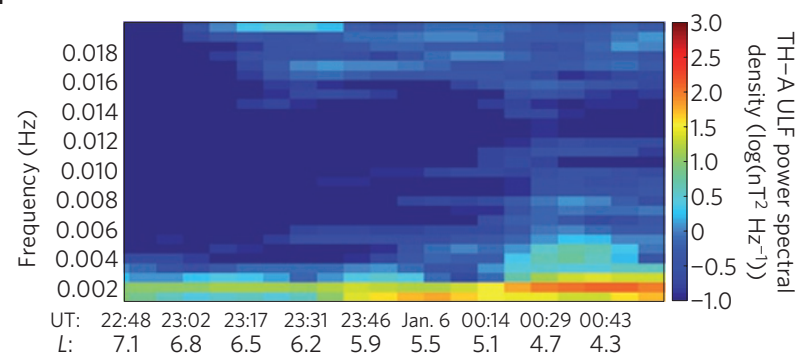

g

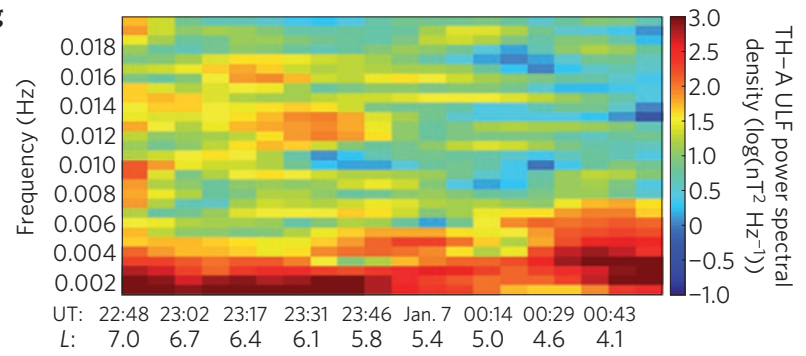

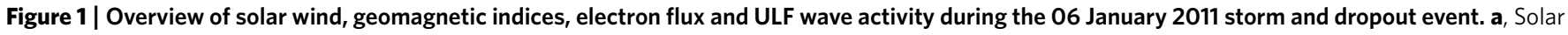
wind dynamic pressure $P_{\text {Dyn }}$ and speed from the OMNI dataset. $\mathbf{b}$, Interplanetary magnetic field (IMF) total field strength $B_{\text {Tot }}$ and $Z$-component in Geocentric Solar Magnetospheric coordinates $B Z$. These exhibit the clear features of the CIR, with the initial compressed slow solar wind, the compressed fast wind segment associated with a strong southward IMF component, and the fast solar wind stream following behind them. The period of negative $B_{Z}$

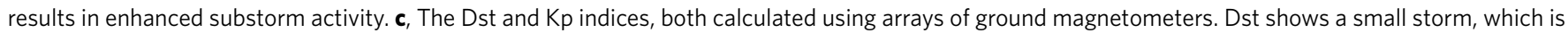
typical for CIRs, with a minimum value of greater than $-50 \mathrm{nT}$, while Kp of 5.7 during the storm's main phase implies very active geomagnetic conditions. d, GOES-13 > $800 \mathrm{keV}$ electron fluxes $\left(\# \mathrm{~cm}^{-2} \mathrm{~s}^{-1} \mathrm{sr}^{-1}\right)$ measured from geosynchronous orbit showing the main phase flux dropout and subsequent recovery phase enhancement. e, McGrath ground station (MCGR) magnetometer data, D-component ( east), with the d.c. field removed (No-d.c.). Enhanced ULF wave activity is evident during the entirety of the storm. The blue shaded periods marked by dashed lines indicate the overlap with the periods shown in $\mathbf{f}$ and $\mathbf{g}$. $\mathbf{f}, \mathbf{g}$, The TH-A power spectral densities for the magnetic field magnitude (d.c. field removed) exhibiting ULF waves at frequencies comparable to outer belt electron drift frequencies. $\mathbf{f}$, The TH-A inbound pass on 05-06 January during the quiet time before the storm. $\mathbf{g}$, The following inbound pass during the main phase and flux dropout.

On 06 January 2011, a co-rotating interaction region (CIR) impacted Earth's magnetosphere, resulting in a small geomagnetic storm, as indicated by the Dst index (minimum $>-50 \mathrm{nT}$; Fig. 1). Associated with the main phase of this storm, several spacecraft from vantage points throughout the outer radiation belt observed a dropout of relativistic electrons (Figs $1 \mathrm{~d}$ and 2e,g). The dropout of radiation belt fluxes occurred over a broad range of energies ( $>300 \mathrm{keV}$ from the National Oceanic and Atmospheric Administration Polar Orbiting Environmental Satellites (NOAA-POES) to $>800 \mathrm{keV}$ from Geostationary Operational Environmental Satellite (GOES)), and fluxes decreased over a range of $L$-shells encompassing almost the entirety of the outer belt. The dropout was clearly non-adiabatic and propagated generally from higher to lower
L-shells based on the GOES observations (Supplementary Figs S1, S15-S17). Furthermore, the dropout did not result from a change in pitch-angle distribution because it was observed over the same range in $L$ by the NOAA-POES spacecraft in Sun-synchronous, low-Earth orbits (measuring low equatorial pitch angles over the full range of the outer belt) and the three THEMIS spacecraft (TH-A, TH-D, and TH-E) in the noon local time sector near the equatorial plane (measuring high equatorial pitch angles throughout the outer belt), as seen in the red lines of Fig. $2 b$ and in Supplementary Fig. S2. Interestingly, based on the NOAA-POES data, the fluxes of $>30 \mathrm{keV}$ and $>100 \mathrm{keV}$ electrons (Fig. 2) did not experience the same dropout. We infer that at those energies the electron losses due to the inward magnetopause motion were more 
a

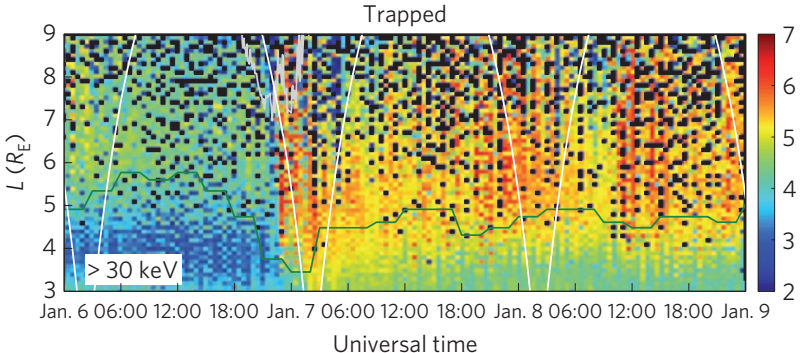

Universal time

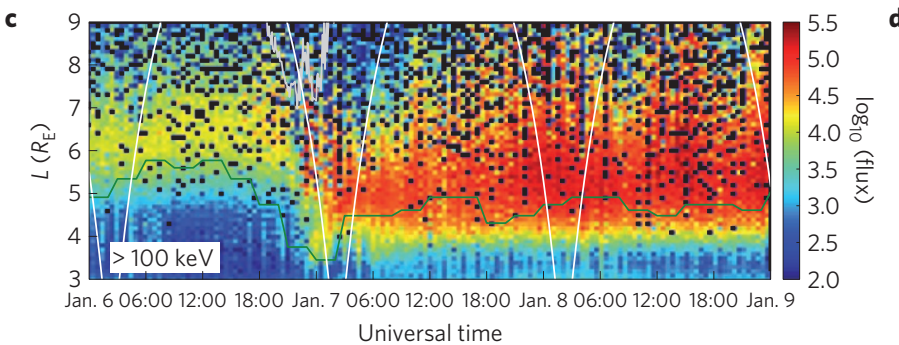

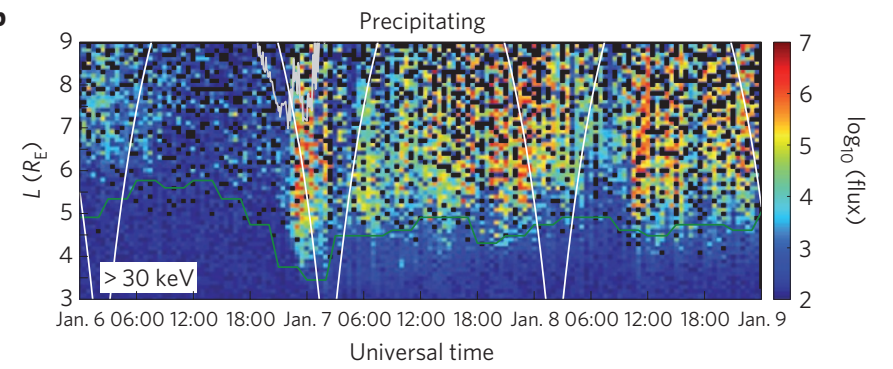

d

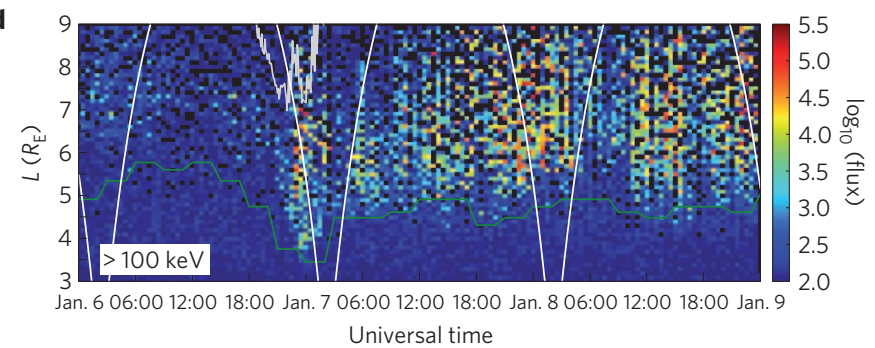

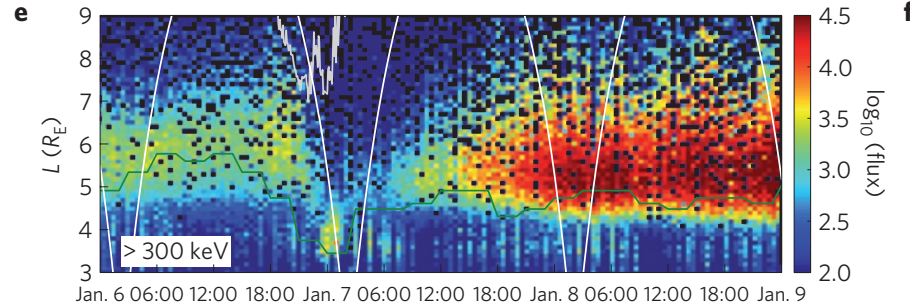

Universal time

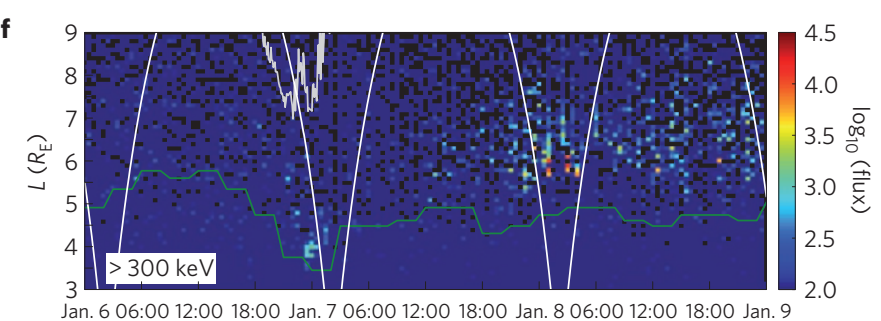

Universal time

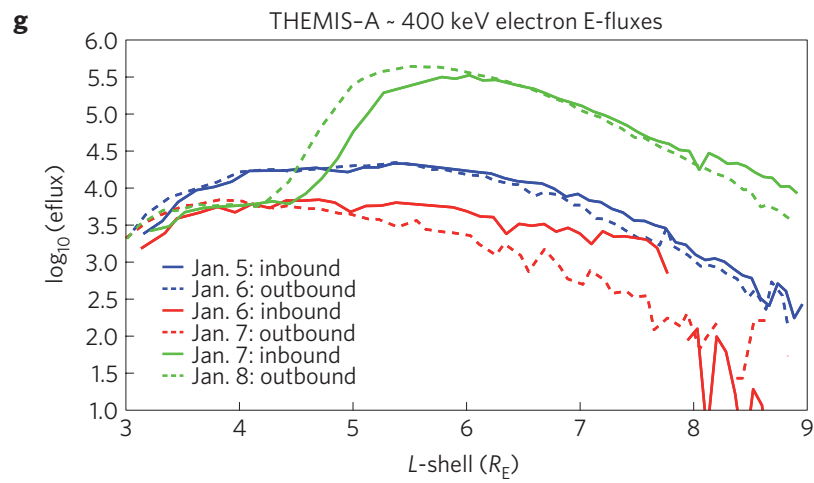

Figure 2 | Electron flux observations from low-Earth orbit and near the magnetic equator during the event. a-f, NOAA-POES Medium Energy Proton/Electron Detector (MEPED) fluxes $\left(\# \mathrm{~cm}^{-2} \mathrm{~s}^{-1} \mathrm{sr}^{-1}\right)$ corrected for proton contamination ${ }^{30}$ and binned by $L$-shell $\left(0.1 R_{\mathrm{E}}\right.$ resolution) and time (30-min resolution). a, $\mathbf{c}$ and $\mathbf{e}$ show the trapped population, while $\mathbf{b}, \mathbf{d}$ and $\mathbf{f}$ show precipitating electrons. The rows show the different electron energy channels as labelled and data gaps are indicated in black. The green line is the plasmapause location ${ }^{31}$, and the grey line is the magnetopause location ${ }^{32}$ White traces show the TH-A orbit tracks, which are very similar to TH-D and TH-E during this period. The dropout at $>300 \mathrm{keV}$ is clearly evident and extends to $L \sim 5$, which is significantly lower than the minimum magnetopause distance of $7 R_{\mathrm{E}}$. Also, the fluxes around $L \sim 4$ are enhanced during the dropout in both the trapped and precipitating populations. This precipitation is observed in the dusk sector in both the Northern and Southern hemispheres. $\mathbf{g}$, TH-A energy fluxes $\left(\mathrm{eV} \mathrm{cm}^{-2} \mathrm{~s}^{-1} \mathrm{sr}^{-1} \mathrm{eV}^{-1}\right)$ from the first Solid State Telescope (SST) coincidence channel, representing electrons with an equivalent energy of $\sim 400 \mathrm{keV}$. This channel is relatively unaffected by proton contamination. The passes shown here correspond to the first six inbound and outbound shown in a-f, and they are colour-coded as indicated in the legend. Note that there is generally good agreement between THEMIS and NOAA observations before, during, and after the dropout.

than balanced by substorm injections and enhanced convection from a source in the plasma sheet; this occurred nearly instantaneously for the $>30 \mathrm{keV}$ electrons and within half a day for the $>100 \mathrm{keV}$ electrons. These injections resulted in an overall enhancement of electrons with these energies throughout the outer belt and increased precipitation of these electrons to the atmosphere. However, the radiation belt electrons with energy $>300 \mathrm{keV}$ exhibited a markedly different behaviour (Fig. 2); their dropout persisted for over $6 \mathrm{~h}$ at $L \sim 5$ and even longer at higher $L$-shells. At the time of the dropout, the $>300 \mathrm{keV}$-precipitating electron fluxes were not enhanced. Such precipitation took place only about a day later, well after the main-phase dropout, when the $>300 \mathrm{keV}$-trapped fluxes increased to levels much higher than before the storm. This is clear evidence that most of the true losses from the system during the main-phase dropout were to the magnetopause. For this to have been the case, outward radial transport must have played a role, as the magnetopause only moved in to $\sim 7 R_{\mathrm{E}}$ during the pressure enhancement associated with the CIR. 
a

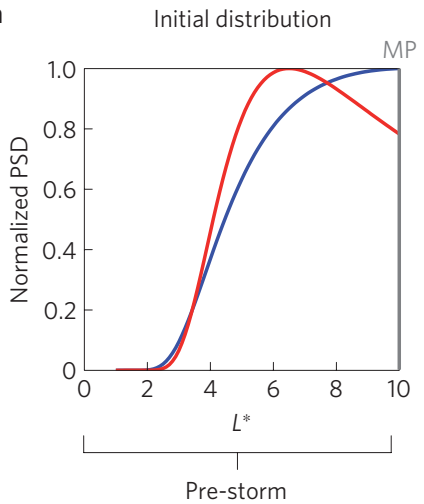

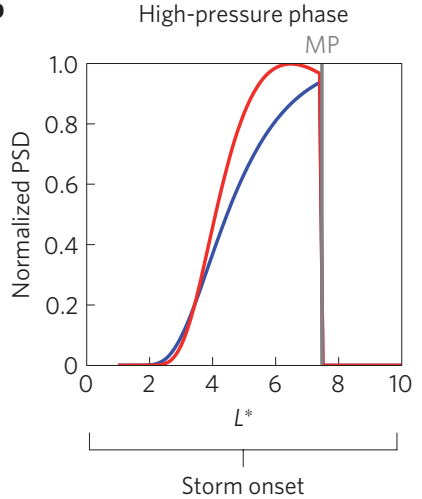
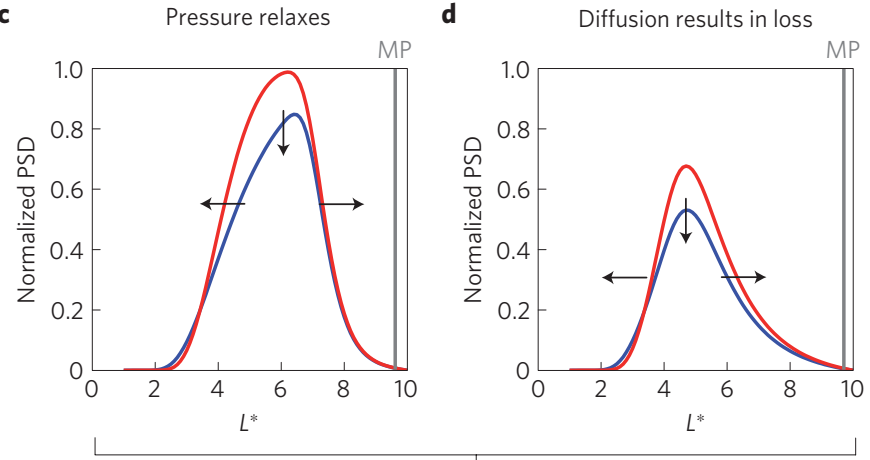

Storm main phase

Figure 3 | Schematic depicting distributions of electron PSD in $L^{*}$ (similar to $L$, but inversely proportional to the third adiabatic invariant and thus also an invariant quantity) for the scenario in which magnetopause shadowing followed by outward radial diffusion results in a dropout of energetic electrons throughout most of the outer belt. a, Before the CIR impacts the magnetosphere, initial distributions for both a positive PSD distribution (blue) and a peaked distribution (red) are shown. $\mathbf{b}$, The high-pressure solar wind at the start of the CIR moves the magnetopause (MP) inward, resulting in magnetopause shadowing. $\mathbf{c}, \mathbf{d}$, The magnetopause moves back out, responding to the lower pressure as the main phase of the storm begins. The loss above $\sim 7 R_{\mathrm{E}}$ results in a very sharp PSD gradient and subsequent enhanced diffusion, as indicated by the black arrows. Outward radial transport results in further losses to the magnetopause and irreversible flux reductions throughout much of the rest of the belt, because electrons lose energy as they move outward. Diffusion coefficients calculated using the global ULF waves observed during the 06 January 2011 event (see Supplementary Information) reveal that rapid outward radial diffusion would have occurred when the combined effects of the enhanced diffusion coefficient and the sharp radial gradient are accounted for. Rapid outward radial transport resulting in flux loss can also occur during the storm's main phase through enhanced electric fields ${ }^{19}$ and/or diamagnetic effects from a partial ring current ${ }^{17}$.

Enhanced ULF wave activity, observed both on the ground and in space, occurred during the main phase of the storm, as seen in Fig. 1e-g. These ULF waves exhibited globally intensified power (Supplementary Figs S18 and S19), when compared with the quiet time before the storm, over a broad band of frequencies comparable to drift rates of $>300 \mathrm{keV}$ electrons and at $L$-shells exhibiting the flux dropout $(L>4)$. ULF waves at these frequencies violated the third adiabatic invariants of the energetic electrons and allowed radial diffusion. The rate of diffusion: (1) increases with ULF wave power ${ }^{26}$, (2) increases with increasing $L$, and (3) is dependent on the PSD gradient. Thus, losses due to magnetopause shadowing are compounded by further loss to the outer boundary through rapid outward radial diffusion, which is driven by the sharp negative gradients in PSD from the initial loss and enhanced ULF wave activity, as explained in Fig. 3. Further rapid outward radial transport may also occur during the storm's main phase owing to enhanced electric fields and/or a partial ring current. Furthermore, any outward radial transport results in a flux decrease at lower $L$-shells throughout the belt because electrons are simultaneously decelerated owing to decreased magnetic field strengths and diverging mirror points as they move outward while conserving their first and second adiabatic invariants. Simulations of these scenarios using test-particles in realistic fields ${ }^{17,19}$ or one-dimensional radial diffusion models (ref. 18; Supplementary Fig. S3) confirm that these mechanisms can indeed deplete the outer radiation belt above $L \sim 4 R_{\mathrm{E}}$ rapidly (in only a few hours).

The results presented here clearly show that the majority of non-adiabatic loss of outer radiation belt electrons with energies above $300 \mathrm{keV}$ during the main phase of a small, CIR-driven geomagnetic storm was not to the atmosphere but to Earth's magnetopause through magnetopause shadowing and subsequent rapid outward radial transport, which further enhanced the total flux loss throughout much of the rest of the belt. This is a viable loss mechanism for outer belt flux dropouts during not only storms but also any event initiated by a period of high solar wind dynamic pressure that results in the magnetopause moving into the outer range of the radiation belt. Interestingly, coronal-mass-injectionand CIR-driven storms often meet this condition, including ones examined in previous studies ${ }^{4,11,23}$, which also reveal that there is insufficient loss to the atmosphere to explain the dropouts (Supplementary Figs S4, S5 and S6). This loss mechanism is also consistent with recent statistical studies that showed many electron dropouts are associated with solar wind dynamic pressure enhancements ${ }^{27}$ and there is no evidence for enhanced electron precipitation during main-phase dropouts in CIR-driven storms ${ }^{28}$. Some important questions remain, however, including: (1) Comparatively, how much do a partial ring current ${ }^{17}$, enhanced outward diffusion $^{18}$, and enhanced electric fields ${ }^{19}$ contribute to the outward radial transport of electrons? (2) What causes loss during dropouts that do not correspond to magnetopause shadowing events?

Non-adiabatic losses during the storm-time main phase have broader implications concerning the extreme dynamics of the outer radiation belt. After flux dropouts such as the ones discussed here, the relativistic electron population is almost entirely depleted and subsequently replenished by a fresh population of electrons from an active source during storm recovery. This is analogous to the outer radiation belt being 'reset' at every storm associated with an onset of high dynamic pressure in the solar wind. Armed with this new understanding, more accurate system models of storm main phases can be developed to better study and understand competition between sources, losses and transport during the recovery phases $^{29}$ and better predict overall storm-time flux variation. This improved understanding of outer radiation belt dynamics will be complemented by the upcoming NASA Radiation Belt Storm Probes (RBSP) mission in conjunction with continuing missions such as GOES, POES and THEMIS.

Received 6 June 2011; accepted 28 November 2011; published online 29 January 2012

\section{References}

1. Van Allen, J. A. \& Frank, L. A. Radiation around the Earth to a radial distance of 107,400 km. Nature 183, 430-434 (1959).

2. Li, X. et al. Are energetic electrons in the solar wind the source of the outer radiation belt? Geophys. Res. Lett. 24, 923-926 (1997).

3. Friedel, R. H. W., Reeves, G. D. \& Obara, T. Relativistic electron dynamics in the inner magnetosphere-a review. J. Atmos. Solar Terr. Phys. 64, 265-282 (2002).

4. Onsager, T. G. et al. Radiation belt electron flux dropouts: Local time, radial, and particle-energy dependence. J. Geophys. Res. 107, 1382 (2002). 
5. Borovsky, J. E. \& Denton, M. H. Relativistic-electron dropouts and recovery: a superposed epoch study of the magnetosphere and the solar wind. J. Geophys. Res. 114, A02201 (2009).

6. Gonzalez, W. D., Tsurutani, B. T. \& Clua de Gonzalez, A. L. Interplanetary origin of geomagnetic storms. Space Sci. Rev. 88, 529-562 (2001).

7. Dessler, A. J. \& Karplus, R. Some effects of diamagnetic ring currents on Van Allen radiation. J. Geophys. Res. 66, 2289-2295 (1961).

8. McIlwain, C. E. Ring current effects on trapped particles. J. Geophys. Res. 71, 3623-3628 (1971).

9. Li, X. et al. Multi-satellite observations of the outer zone electron variation during the Nov. 3-4 1993 magnetic storm. J. Geophys. Res. 102, 14123-14140 (1997).

10. Kim, H-J. \& Chan, A. A. Fully adiabatic changes in storm-time relativistic electron fluxes. J. Geophys. Res. 102, 22107-22116 (1997).

11. Green, J. C. et al. Testing loss mechanisms capable of rapidly depleting relativistic electron flux in the Earth's outer radiation belt. J. Geophys. Res. 109, A12211 (2004).

12. Millan, R. \& Thorne, R. M. Review of radiation belt relativistic electron losses. J. Atmos. Solar Terr. Phys. 69, 362-377 (2007).

13. Millan, R. et al. Observation of relativistic electron precipitation during a rapid decrease of trapped relativistic electron flux. Geophys. Res. Lett. 34, L10101 (2007)

14. Sergeev, V. A. \& Tsyganenko, N. A. Energetic particle losses and trapping boundaries as deduced from calculations with a realistic magnetic field model. Planet. Space Sci. 30, 999-1006 (1982).

15. Horne, R. B., Lam, M. M. \& Green, J. C. Energetic electron precipitation from the outer radiation belt during geomagnetic storms. Geophys. Res. Lett. 36, L19104 (2009).

16. Sandanger, M. I. et al. Relativistic electron losses related to EMIC waves during CIR and CME storms. J. Atmos. Solar Terr. Phys. 71, 1126-1144 (2009).

17. Ukhorskiy, A. Y. et al. Storm time evolution of the outer radiation belt: Transport and losses. J. Geophys. Res. 111, A11S03 (2006).

18. Shprits, Y. Y. et al. Outward radial diffusion driven by losses at magnetopause. J. Geophys. Res. 111, A11214 (2006).

19. Nishimura, Y. et al. Evolution of ring current and radiation belt particles under the influence of storm-time electric fields. J. Geophys. Res. 112, A06241 (2007).

20. Chen, Y., Reeves, G. D. \& Friedel, R. H. W. The energization of relativistic electrons in the Van Allen radiation belt. Nature Phys. 3, 614-617 (2007).

21. Turner, D. L. et al. On phase space density radial gradients of Earth's outer-belt electrons prior to sudden solar wind pressure enhancements: Results from distinctive events and a superposed epoch analysis. J. Geophys. Res. 115, A01205 (2010)

22. Hudson, M. K. et al. Increase in relativistic electron flux in the inner magnetosphere: ULF wave mode structure. Adv. Space Res. 25, 2327-2337 (2000)
23. Loto'aniu, T. M. et al. Relativistic electron loss due to ultralow frequency waves and enhanced outward radial diffusion. J. Geophys. Res. 115, A12245 (2010).

24. Bortnik, J. et al. Observation of two distinct, rapid loss mechanisms during the 20 Nov. 2003 radiation belt dropout event. J. Geophys. Res. 111, A12216 (2006).

25. Millan, R. M. et al. Spatial distribution of relativistic electron precipitation during a radiation belt depletion event. Geophys. Res. Lett. 37, L20103 (2010)

26. Mathie, R. A. \& Mann, I. R. A correlation between extended intervals of ULF wave power and storm-time geosynchronous relativistic electron flux enhancements. Geophys. Res. Lett. 27, 3261-3264 (2000).

27. Morley, S. K. et al. Dropouts of the outer electron radiation belt in response to solar wind stream interfaces: Global positioning system observations. Proc. R. Soc. A 466, 3329-3350 (2010).

28. Meredith, N. P. et al. Energetic electron precipitation during high-speed solar wind stream driven storms. J. Geophys. Res. 116, A05223 (2011).

29. Reeves, G. D. et al. Acceleration and loss of relativistic electrons during geomagnetic storms. Geophys. Res. Lett. 30, 1529 (2003).

30. Lam, M. M. et al. Origin of energetic electron precipitation $>30 \mathrm{keV}$ into the atmosphere. J. Geophys. Res. 115, A00F08 (2010).

31. O’Brien, T. P. \& Moldwin, M. B. Empirical plasmapause models from magnetic indices. Geophys. Res. Lett. 30, 1152 (2003).

32. Shue, J. H. et al. A new functional form to study the solar wind control of the magnetopause size and shape. J. Geophys. Res. 102, 9497-9511 (1997).

\section{Acknowledgements}

We thank J. Green and J. Rodriguez for providing details concerning the NOAA-POES and GOES-Magnetospheric Electron Detector (MAGED) data, respectively, and for useful comments. Thanks to the NOAA, THEMIS (D. Larson and R. P. Lin for the SST data, K. H. Glassmeier, U. Auster, and W. Baumjohann for the flux gate magnetometer data, and the University of Alaska for the ground-based magnetometer data), OMNI, and Kyoto World Data Center for Geomagnetism (Kyoto-WDC) teams for their online data access. This work was supported by NASA contract NAS5-02099.

\section{Author contributions}

D.L.T. conducted the majority of the data processing and analysis and writing for this study. Y.S. suggested to simultaneously study precipitating and trapped data and assisted with data interpretation and the diffusion simulations. M.H. analysed the THEMIS ULF wave data and provided input on ULF waves and activity. V.A. assisted with data interpretation and provided the THEMIS-SST data.

\section{Additional information}

The authors declare no competing financial interests. Supplementary information accompanies this paper on www.nature.com/naturephysics. Reprints and permissions information is available online at www.nature.com/reprints. Correspondence and requests for materials should be addressed to D.L.T. 